\title{
High frequencies of polyfunctional HIV-specific T cells are associated with preservation of mucosal CD4 T cells in bronchoalveolar lavage
}

\author{
JM Brenchley ${ }^{1,7}$, KS Knox ${ }^{2,3,7}$, AI Asher ${ }^{1}$, DA Price ${ }^{1,4}$, LM Kohli $^{2}$, E Gostick ${ }^{4}$, BJ Hill1 ${ }^{1}$, CA Hage ${ }^{2,3}$, \\ Z Brahmi $^{5}$, A Khoruts ${ }^{6}$, HL Twigg III ${ }^{2}$, TW Schacker ${ }^{6}$ and DC Douek ${ }^{1}$
}

The mechanisms underlying the massive gastrointestinal tract CD4 T-cell depletion in human immunodeficiency virus (HIV) infection are not well understood nor is it clear whether similar depletion is manifest at other mucosal surfaces. Studies of T-cell and virus dynamics in different anatomical sites have begun to illuminate the pathogenesis of HIVassociated disease. Here, we studied depletion and HIV infection frequencies of CD4 T cells from the gastrointestinal tract, bronchoalveolar lavage (BAL), and blood with the frequencies and functional profiles of HIV-specific T cells in these anatomically distinct sites in HIV-infected individuals. The major findings to emerge were as follows: (i) depletion of gastrointestinal CD4 T cells is associated with high frequencies of infected CD4 T cells; (ii) HIV-specific T cells are present at low frequencies in the gastrointestinal tract compared to blood; (iii) BAL CD4 T cells are not massively depleted during the chronic phase; (iv) infection frequencies of BAL CD4 T cells are similar to those in blood; (v) significantly higher frequencies and increased functionality of HIV-specific T cells were observed in BAL compared to blood. Taken together, these data suggest mechanisms for mucosal CD4 T-cell depletion and interventions that might circumvent global depletion of mucosal CD4 T cells.

\section{INTRODUCTION}

Massive depletion of gastrointestinal CD4 T cells is a hallmark of human immunodeficiency virus (HIV) and simian immunodeficiency virus (SIV) infection. ${ }^{1-6}$ This depletion occurs during the acute phase of infection and is maintained throughout the chronic phase. The mechanism underlying this depletion was recently shown to be the direct consequence of target-cell infection ${ }^{4}$ and virus-induced Fas-mediated apoptosis; ${ }^{5}$ however, such studies in HIV infection are scarce. Moreover, while it is clear that substantial depletion of CD4 T cells occurs in the gastrointestinal (GI) tract of HIV-infected individuals, it is unclear if similar depletion is manifest in other mucosal lymphoid sites.

The role of HIV-specific T-cell immunity in protecting against mucosal CD4 T-cell depletion is unclear in light of these recent advances. There are multiple lines of evidence to suggest that HIV-specific T cells play an important role in the control of viral replication in peripheral blood ${ }^{7-11}$ and that HIV-specific T cells can be detected in mucosal sites. ${ }^{12-14}$ Furthermore, while SIVspecific CD8 T cells are associated with preservation of central memory CD4 T cells following vaccination and subsequent SIV challenge, ${ }^{15-17}$ the complex interrelationships between adaptive T-cell immunity, viral infection, and CD4 T-cell depletion from mucosal sites are unclear.

Based on the rationale that observed differences between individual mucosal sites might illuminate mechanisms for protection of memory CD4 T cells, we specifically sampled bronchoalveolar lavage (BAL) and terminal ileum, as these two anatomically distant compartments are both predominantly "effector" lymphoid tissues. Populations of lung cells obtained by BAL and GI tract

\footnotetext{
${ }^{1}$ Human Immunology Section, Vaccine Research Center, National Institute of Allergy and Infectious Diseases, National Institutes of Health, Bethesda, Maryland, USA. ${ }^{2}$ Division of Pulmonary and Critical Care Medicine, Indiana University, Indianapolis, Indiana, USA. ${ }^{3}$ Richard L Roudebush VA Medical Center, Indianapolis, Indiana, USA. ${ }^{4}$ Weatherall Institute of Molecular Medicine, University of Oxford, Oxford, UK. ${ }^{5}$ Department of Medicine, Indiana University Medical Center, Indianapolis, Indiana, USA. ${ }^{6}$ Department of Medicine, University of Minnesota, Minneapolis, Minnesota, USA. ${ }^{7}$ These authors contributed equally to this work. Correspondence: DC Douek or JM Brenchley (ddouek@mail.nih.gov) 
cells in the lamina propria are similar in that they lack naive $\mathrm{T}$ cells. Moreover, BAL and gastrointestinal lymphocytes are exposed to the external environment and thus represent distinct mucosal sites. ${ }^{18}$

In this study, we recruited 26 treatment-naive, HIV-infected individuals at different stages of disease and $12 \mathrm{HIV}$-uninfected individuals. Biopsies of terminal ileum $\left(13 \mathrm{HIV}^{+}\right.$and $\left.6 \mathrm{HIV}^{-}\right)$ or BALs $\left(13 \mathrm{HIV}^{+}\right.$and $\left.6 \mathrm{HIV}^{-}\right)$were obtained from separate cohorts (Table 1). All subjects had blood analyzed for comparison to their respective tissue compartment. From each subject and within each compartment, we examined (i) CD4 T-cell depletion; (ii) relative frequencies of infected memory CD4 T cells; and (iii) the frequency and functional capacity of HIVspecific CD4 and CD8 T cells.

\section{RESULTS Depletion of gastrointestinal CD4 T cells in GI tract and BAL}

To determine the relative depletion of $\mathrm{CD} 4 \mathrm{~T}$ cells from mucosal sites compared to blood, we first determined the frequency of $\mathrm{T}$ cells that expressed CD4 (Figure 1a,b) from matched terminal ileum and blood samples as well as matched BAL and blood samples. As both BAL and terminal ileum contained lower frequencies of $\mathrm{CD} 4 \mathrm{~T}$ cells compared to blood, these data would at first appear to suggest that $\mathrm{CD} 4 \mathrm{~T}$ cells are, indeed, preferentially depleted in both GI tract and BAL. However, we have previously demonstrated that simple analysis of CD4 T-cell frequencies cannot, reliably, be used to assess preferential depletion of CD4 T cells from mucosal sites compared to peripheral blood as CD4 to CD8 ratios are inverted in the terminal ileum of the GI tract even in the absence of HIV infection. ${ }^{2}$ Moreover, CD4 to CD8 ratios in BAL are notably sensitive to factors such as smoking, ${ }^{19}$ hypersensitivity pneumonitis, ${ }^{20}$ and viral respiratory infections. ${ }^{21}$ Indeed, of our BAL subjects, 8 out of 10 of the HIV-infected individuals while only 1 out of 5 of the HIV-uninfected individuals were regular cigarette smokers. Hence, to determine more rigorously the relative depletion of CD4 T cells from the terminal ileum and BAL, we measured the frequency of $\mathrm{CCR} 5^{+}$memory $\mathrm{CD} 4 \mathrm{~T}$ cells, the cellular target for HIV, in blood (Figure 1c), GI tract (Figure 1d), and BAL (Figure 1e). Consistent with previous reports, we found almost complete depletion of CCR ${ }^{+} \mathrm{CD} 4 \mathrm{~T}$ cells from the GI tract of HIV-infected individuals compared to HIV-uninfected individuals (Figure 1d). ${ }^{2,3}$ This depletion of CCR $5^{+}$memory CD4 T cells was evident in all HIV-infected individuals irrespective of the disease state. However, in stark contrast, we found no obvious depletion of CCR $5{ }^{+} \mathrm{CD} 4 \mathrm{~T}$ cells from BAL of HIVinfected individuals compared to uninfected individuals (Figure 1e). In fact, we found no difference in the frequencies of BAL memory CCR $5^{+} \mathrm{CD} 4 \mathrm{~T}$ cells between our infected and uninfected cohorts. Moreover, the vast majority of CD8 T cells from both BAL and terminal ileum of HIV-infected individuals consistently express CCR5 (data not shown). These data suggest that the pulmonary mucosal site represented by BAL appears to evade the massive $\mathrm{CD} 4 \mathrm{~T}$-cell depletion characteristic of the GI tract.

\section{Infection frequency of CD4 T cells from blood, BAL, and terminal ileum}

Recent analyses of acutely SIV-infected rhesus macaques have demonstrated that the majority of CD4 T-cell depletion that occurs in the GI tract is the consequence of direct viral infection, as very high frequencies of infected CD4 $\mathrm{T}$ cells are observed at this site during acute infection. ${ }^{4,5}$ To determine whether infection frequency of mucosal CD4 T cells could account for depletion of mucosal CD4 T cells in chronically HIV-infected humans, we sorted memory CD4 $\mathrm{T}$ cells from blood, terminal ileum, and BAL, and subjected these cells to quantitative PCR (qPCR) for HIV gag DNA. As naive CD4 T cells are less frequently infected than memory CD4 $\mathrm{T}$ cells, and since very few naive $\mathrm{CD} 4 \mathrm{~T}$ cells reside at mucosal surfaces, we specifically sorted peripheral blood $\mathrm{CD} 27{ }^{+} \mathrm{CD} 45 \mathrm{RO}{ }^{+} \mathrm{CD} 4 \mathrm{~T}$ cells, as these cells represent the most frequently infected blood $\mathrm{T}$ cell subset in vivo. ${ }^{22}$ Initially, we compared the frequency of infected memory CD4 T cells between blood and the terminal ileum, and found that the memory CD4 $\mathrm{T}$ cells residing in the terminal ileum were, on average, 10 -fold more frequently infected (Figure 2a). These data strongly suggest that the massive depletion observed in GI tract, and at all stages of infection, can be primarily ascribed to the consequences of direct viral infection. Similar comparative analyses were performed on matched blood and BAL samples (Figure $\mathbf{2 b}$ ). In contrast to memory CD4 T cells that reside in GI tract, BAL memory CD4 T cells, while certainly capable of being infected in vivo, were not preferentially targeted by the virus when compared to blood memory CD4 T cells. Moreover, we found a lower viral load (VL) within BAL fluid (BALF) compared to plasma (Table 1). However, several studies have suggested that the major target for viral replication in BAL in vivo, and a significant reservoir of virus, is the alveolar macrophage (AM). ${ }^{23-25}$ Hence, in 10 subjects, we simultaneously sorted memory CD4 T cells and AM from BAL to a purity of $>99 \%$ and analyzed infection frequency by qPCR. Surprisingly, we found only very low copies of viral DNA within AM (Figure 2b). In 3 out of 10 individuals, we found no copies of viral DNA, and in 7 out of 10 individuals, we found less than $0.1 \%$ of AM harboring HIV DNA (median 0.01\%) (Figure 2b). Indeed, as this cell subset is notoriously difficult to sort with high purity, it is impossible to determine whether or not the few copies of viral DNA amplified in 7 out of 10 of the individuals were, in fact, derived from AM or whether the viral DNA was within contaminating $\mathrm{T}$ cells. Thus, these data demonstrate that while AM may be productively infected by HIV in vitro, they are only rarely, if ever, infected in vivo. These data further suggest that while AM may bind to and transfer virions, they are not a substantial reservoir for HIV. To establish a mechanistic rationale for the low infection frequencies of AM, we viably sorted AM and BAL T cells from three individuals, as well as blood monocytes and blood T cells, and performed quantitative RT-PCR for CCR5 mRNA and normalized expression based upon qRT-PCR for hGus (Figure 2c). In all three individuals, the amount of CCR 5 mRNA in AM was only $25 \%$ of the CCR 5 mRNA found in 
Table 1 Subject cohort

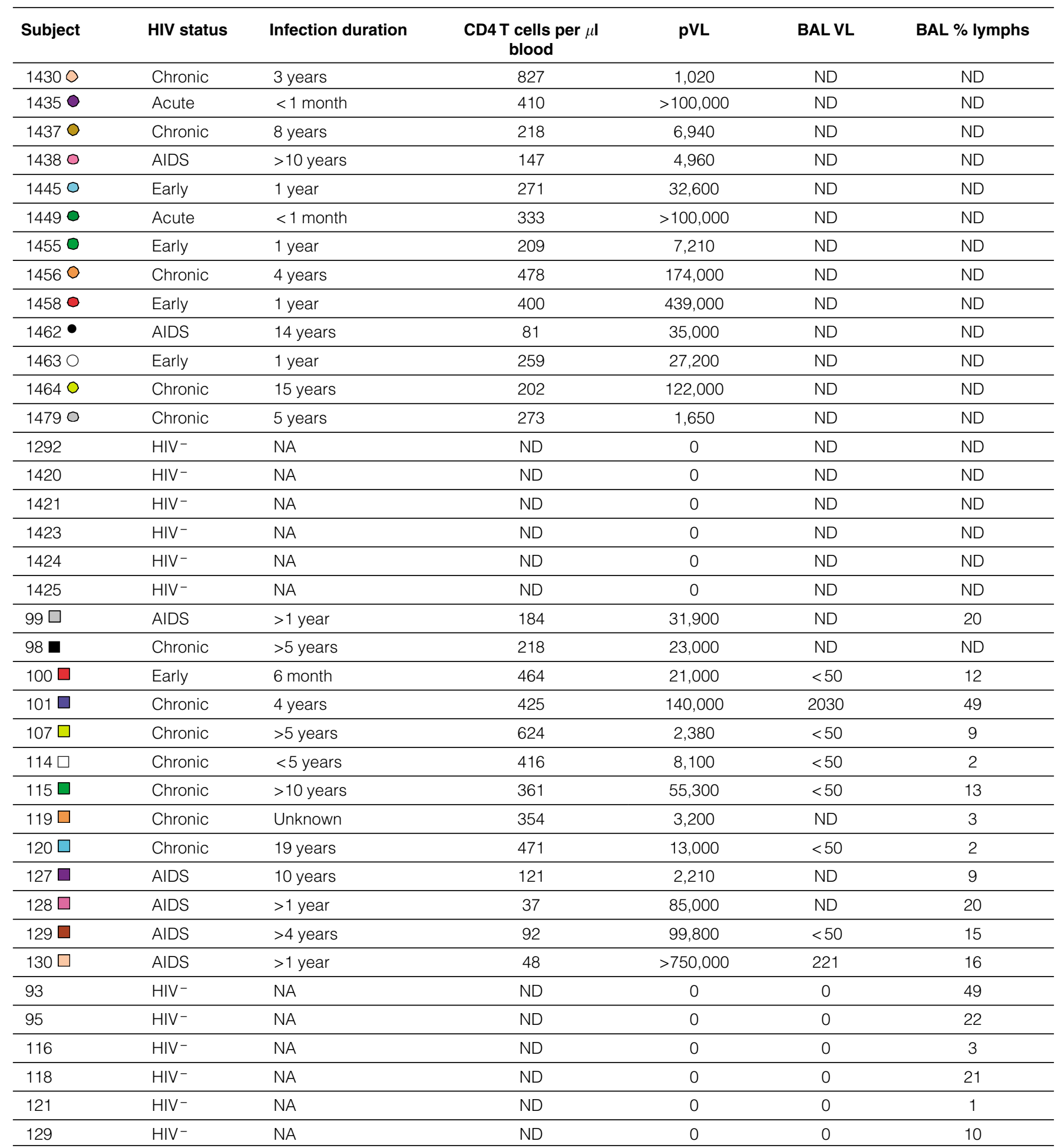

BAL, bronchoalveolar lavage; $p V L$, plasma viral load; NA, not applicable; ND, not determined.

BAL T cells, while blood monocytes expressed $7 \times$ higher levels of CCR 5 mRNA compared to blood T cells (data not shown). Hence AM may be less susceptible to infection in vivo due to their low CCR5 expression.
Frequency and functionality of HIV-specific T cells in the terminal ileum and BAL

Recent data have suggested that highly functional HIV-specific T-cell responses are associated with preservation of peripheral 

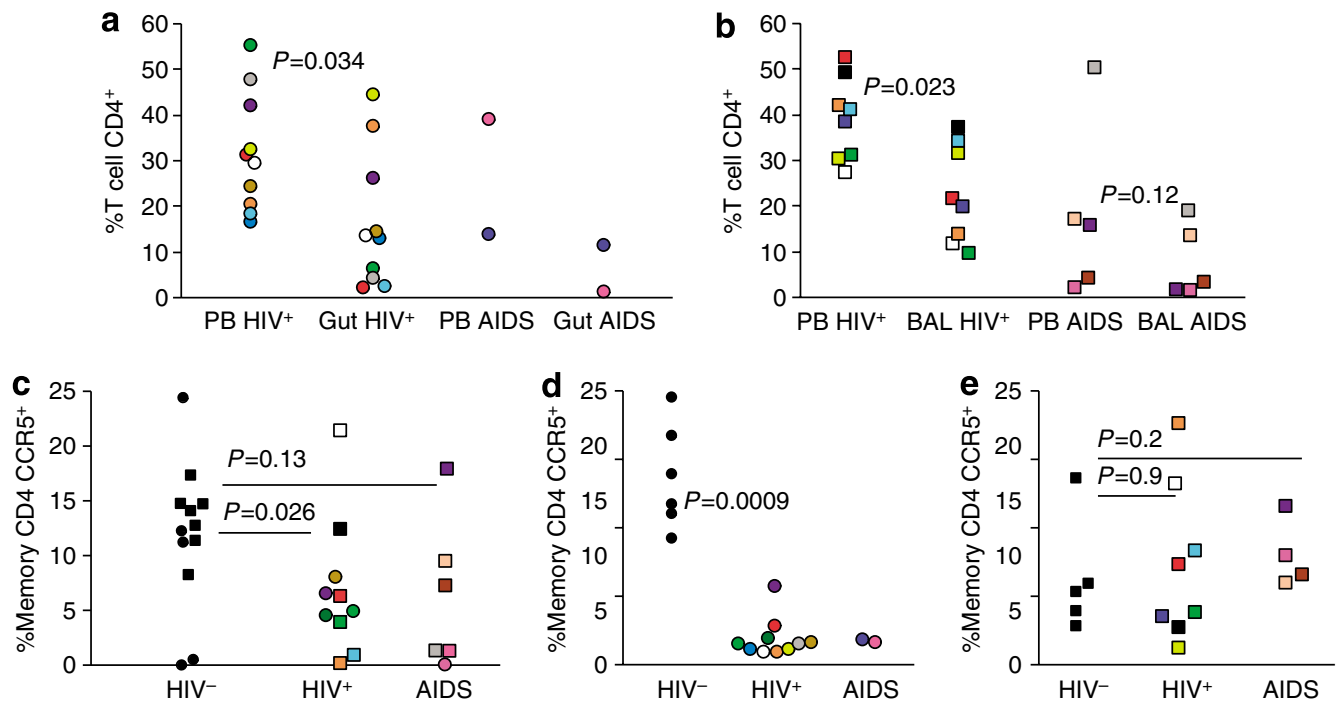

Figure 1 Depletion of CD4 T cells from blood, GI tract, and BAL. (a, b) Percentage of T cells that express CD4 was measured after lymphocyte and CD3 gating in blood, GI tract, and BAL from individuals with chronic HIV infection or AIDS. (c) Percentage of blood memory CD4 T cells that express CCR5 was measured in uninfected, chronically HIV-infected individuals, and individuals with AIDS. (d) Percentage of GI tract memory CD4 T cells that express CCR5 was measured in uninfected, chronically HIV-infected individuals, and individuals with AIDS. (e) Percentage of BAL memory CD4 T cells that express CCR5 was measured in uninfected, chronically HIV-infected individuals, and individuals with AIDS. $P$-values represent the results of Wilcoxon's matched pairs test $(\mathbf{a}, \mathbf{b})$ or Mann-Whitney U-test $(\mathbf{c}, \mathbf{d})$. BAL, bronchoalveolar lavage; GI, gastrointestinal.
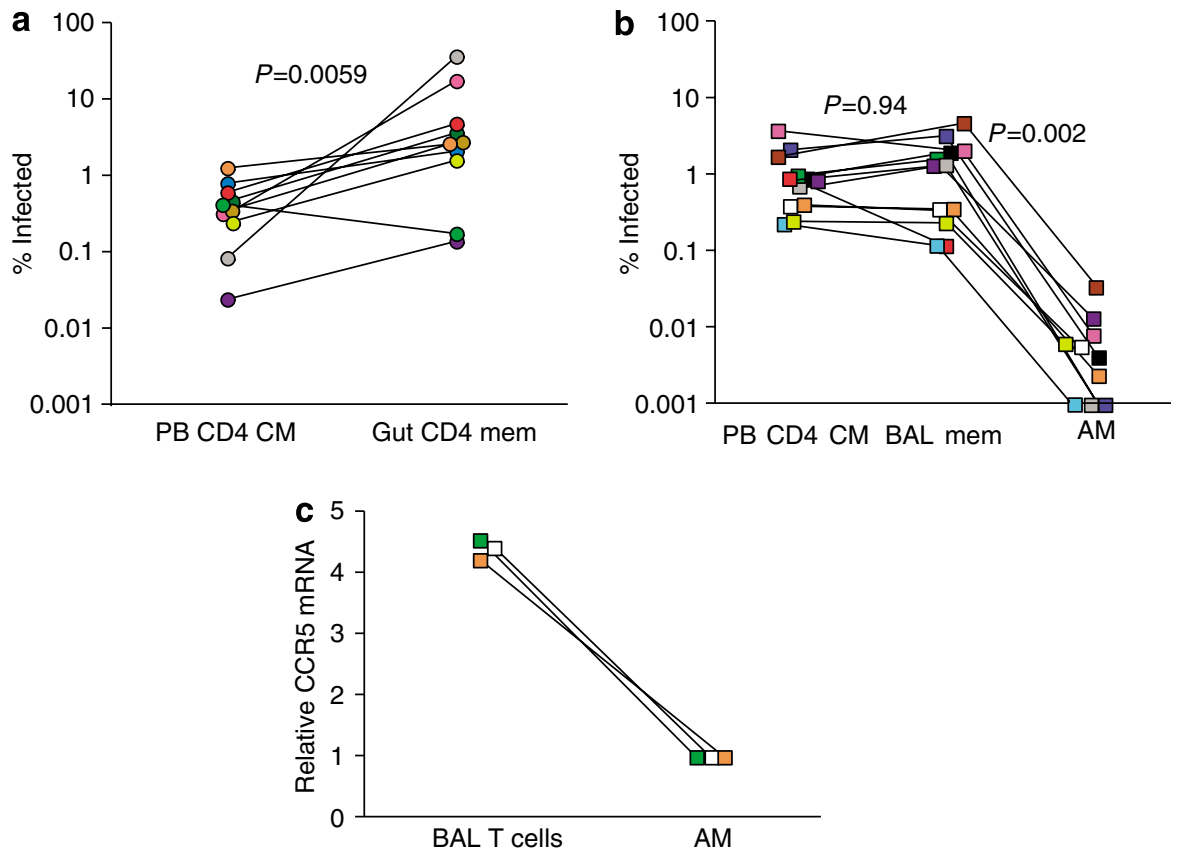

Figure 2 HIV infection frequency of CD4 T cells and alveolar macrophages in blood, GI tract, and BAL. (a) Percentage of HIV-infected blood central memory CD4 T cells and gut memory CD4 T cells determined by qPCR for HIV gag DNA. (b) Percentage of HIV-infected blood central memory CD4 T cells, BAL memory CD4 T cells, and BAL macrophages. (c) The ratio of CCR5 mRNA in BAL memory CD4 T cells compared to BAL macrophages in three individuals. Points depicting values for the same individual are linked by a line. BAL, bronchoalveolar lavage; GI, gastrointestinal.

blood CD4 T cells. ${ }^{26}$ As we observed two disparate immunological outcomes at mucosal surfaces, we studied the frequency and functionality of HIV-specific T cells in blood, BAL, and terminal ileum. Representative data of mitogenically stimulated terminal ileum $\mathrm{T}$ cells from subject 1464 and the flow cytometric gating strategy are demonstrated in Figure 3. After gating for live, memory $\mathrm{CD} 4$ or $\mathrm{CD} 8 \mathrm{~T}$ cells, these data demonstrate that the $\mathrm{T}$ cells from the terminal ileum were capable of producing interferon (IFN) $\gamma$, tumor necrosis factor (TNF), and interkeukin (IL)-2.

Using intracellular cytokine staining for IFN $\gamma$, TNF, and IL-2 following HIV peptide stimulation (Figure 4a) or tetramer staining (Figure 4b), HIV-specific CD8 T cells could be detected in both the terminal ileum and peripheral blood of most indi- 


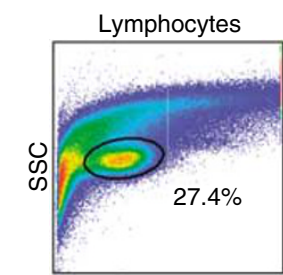

FSC
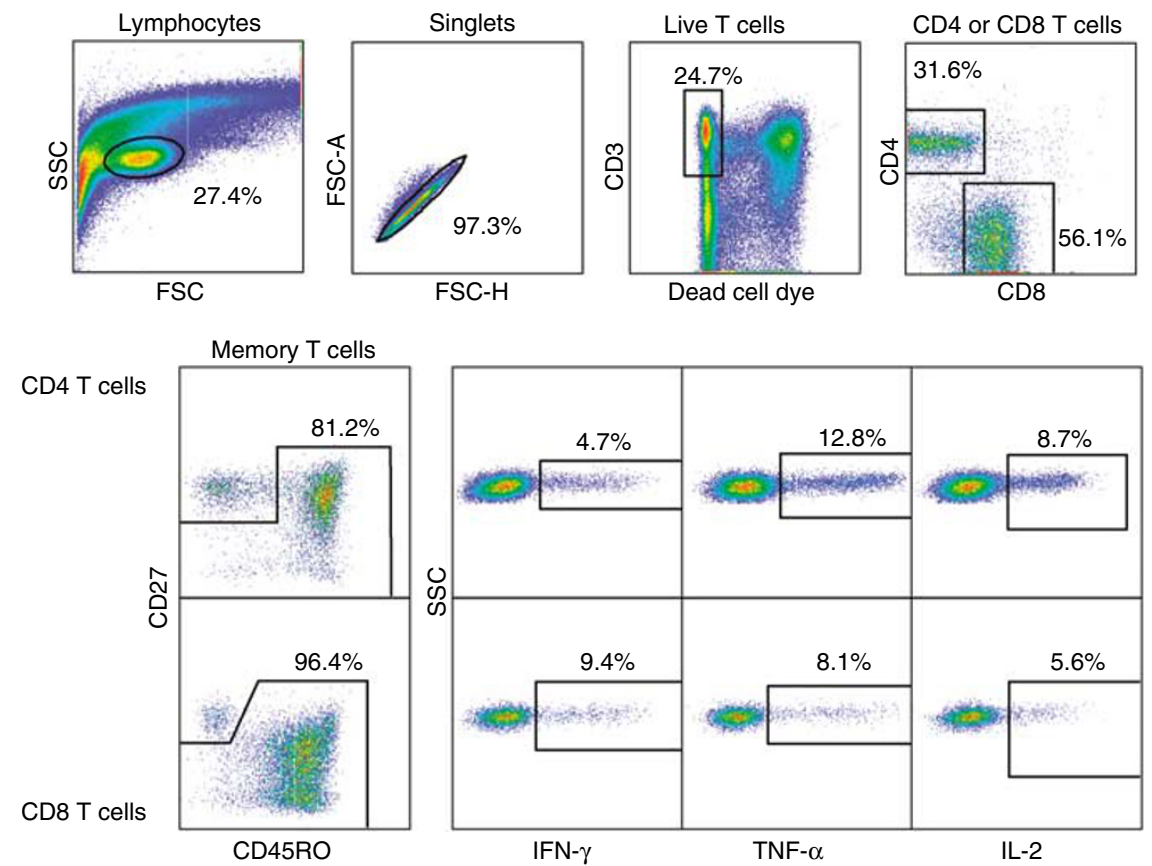

Figure 3 Flow cytometric analysis of cytokine production after antigenic stimulation. GI tract lymphocytes from subject 1464 were stimulated with SEB in the presence of brefeldin A for $18 \mathrm{~h}$ and then stained for surface and intracellular proteins as described in Methods. Lymphocytes are gated based on characteristic light-scatter properties, single lymphocytes are gated based on forward scatter height vs. forward scatter area, and live T cells are gated based on expression of CD3 without staining for the dead cell dye vivid. CD4 or CD8 T cells are then gated and memory CD4 or CD8 T cells are gated based on the characteristic expression patterns of CD27 and CD45RO. The percentage of memory CD4 and CD8 T cells that produce IFN- $\gamma$, TNF, and IL-2 can then be determined. GI, gastrointestinal.

viduals. However, the frequencies of memory CD8 T cells in the GI tract that recognize HIV antigens appeared to be lower than the frequencies of memory CD8 $\mathrm{T}$ cells in blood that recognize the same antigens (Figure 4). Indeed, further analysis revealed significantly lower frequencies of HIV-specific CD8 T cells and no detectable HIV-specific CD4 T cells in the terminal ileum compared to peripheral blood (Figure 5a,b). In addition, three individuals from whom GI tract biopsies were taken had HLA types amenable to analysis of HIV-specific CD8 T cells by pMHC-I tetrameric complexes (Figure $\mathbf{4 b}$ and triangles, Figure 5a). Here, HIV-specific CD8 T cells were physically present in the terminal ileum of only one out of three individuals and even then were present at lower frequencies in the terminal ileum compared to blood.

A markedly different picture emerged upon comparison of HIV-specific T-cell responses in blood and BAL (Figure 5c,d). While both HIV-specific CD8 and CD4 T cells were present at significantly lower frequencies in the terminal ileum compared to blood (Figure 5a,b), HIV-specific T cells were present at high frequencies in BAL (Figure 5c,d). Indeed, we found no significant differences between the frequency of memory CD8 T cells capable of responding to individual HIV antigens between BAL and blood (Figure 5c). In addition, since we simultaneously measured expression of IFN $\gamma$, TNF, and IL2, we were able to determine the relative breadth of functionality of HIV-specific $\mathrm{T}$ cells in blood and BAL. ${ }^{26} \mathrm{HIV}$-specific CD8 $\mathrm{T}$ cells in BAL tended toward a more polyfunctional phenotype compared to blood CD8 T cells (Figure 5e), similar to the functionality of blood HIV-specific CD8 T cells from long-term nonprogres- sors. ${ }^{26}$ Moreover, HIV-specific CD4 T cells from BALs were present at significantly higher precursor frequencies and manifested a significantly more polyfunctional response compared to HIV-specific CD4 T cells from blood (Figure 5d,f). These data are consistent with a role for polyfunctional HIV-specific $\mathrm{T}$ cells in protection against direct infection and subsequent depletion of mucosal CD4 T cells.

One potential mechanism by which HIV-specific T cells may decrease infection of BAL CD4 T cells, compared to GI tract CD4 T cells, would be by the production of local chemokines that bind and lead to internalization of CCR5. Hence, we measured levels of MIP- $1 \alpha$ and MIP- $1 \beta$ in BALF (chemokines quantified in BALF were corrected for dilution based on the estimated volume of epithelial lining fluid as previously described ${ }^{27}$ ) and plasma from subjects in our cohort. In doing so, we found significantly higher concentrations of these chemokines in BALF compared to plasma (Figure 6). Levels of these chemokines were not different when comparing BALF or plasma among $\mathrm{HIV}^{-}$ and $\mathrm{HIV}^{+}$individuals, thereby suggesting that the lung may be uniquely protected from mucosal CD4 T-cell depletion (data not shown). This interpretation is consistent with earlier studies showing that these levels of MIP- $1 \alpha$ and MIP- $1 \beta$ prevent in vitro infections of CD4 T cells with HIV. ${ }^{28}$ In addition to the high frequencies of AM in BAL, a predominant source of MIP- 1 chemokines in the body are CD8 T cells. Indeed, any one CD8 $\mathrm{T}$ cell is capable of producing approximately $0.5 \mathrm{pg}$ of MIP- $1 \beta .{ }^{29}$ Moreover, it is clear that MIP- $1 \beta$ is a predominant factor produced by HIV-specific CD8 T cells. ${ }^{26} \mathrm{To}$ confirm that HIV-specific $\mathrm{T}$ cells produced CCR5-binding chemokines 

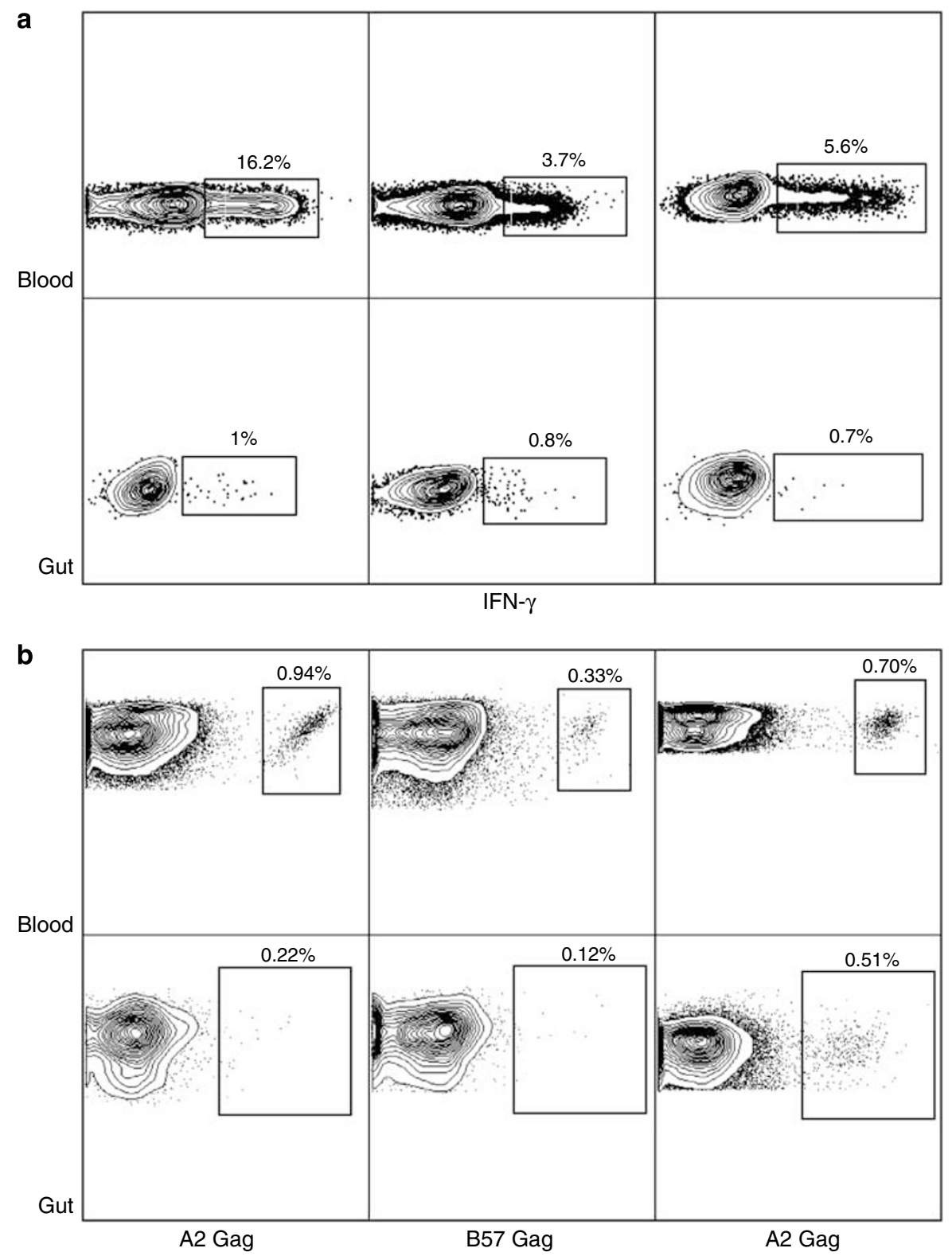

Figure 4 HIV-specific CD8 T cells in blood and GI tract. (a) HIV-specific CD8 T-cell responses in blood and gut defined functionally. (b) HIV-specific CD8 T cells in blood and gut defined physically. Gl tract and peripheral blood lymphocytes from subjects 1458 , 1479, and 1456 were stimulated with overlapping HIV peptides and stained as described in Methods. The responses are shown as the percentage of memory T cells that are HIV-specific (a). Alternatively, tetramer analysis was performed on GI tract and peripheral blood lymphocytes from subjects 1430, 1458, and 1469, and the frequencies of memory CD8 T cells that bind HIV tetramers are shown (b). GI, gastrointestinal.

in response to antigen-specific stimulation, we used intracellular cytokine staining for IFN $\gamma$, TNF, IL2, and MIP-1 $\beta$ after stimulation of BAL and blood lymphocytes from subjects $100,101,115,119$, and 127. Consistent with studies of blood HIV-specific CD8 T cells, ${ }^{26} \mathrm{HIV}$-specific T cells from BAL unanimously produced MIP-1 $\beta$ (Figure 7 ), thus suggesting a role for HIV-specific T cells in protecting against mucosal depletion. Moreover, previous studies have demonstrated that certain lung surfactants can bind to HIV envelope and inhibit infection in vitro. ${ }^{30}$ Hence preservation of BAL CD4 T cells is likely multifaceted.

\section{DISCUSSION}

Here, we investigated viral infection, HIV-specific T-cell immunity, and CD4 T-cell depletion from two anatomically distinct mucosal sites and compared them to blood. Four major points emerged: (i) depletion of GI tract CD4 T cells is associated with very high frequencies of HIV-infected CD4 T cells; (ii) terminal ileum CD4 T-cell depletion and high infection frequencies are associated with low frequencies of HIV-specific T-cell responses within the terminal ileum; (iii) in contrast, BAL CD4 T cells persist and are infected at low frequencies, similar to blood CD4 T cells; and (iv) high frequencies of mucosal polyfunctional 

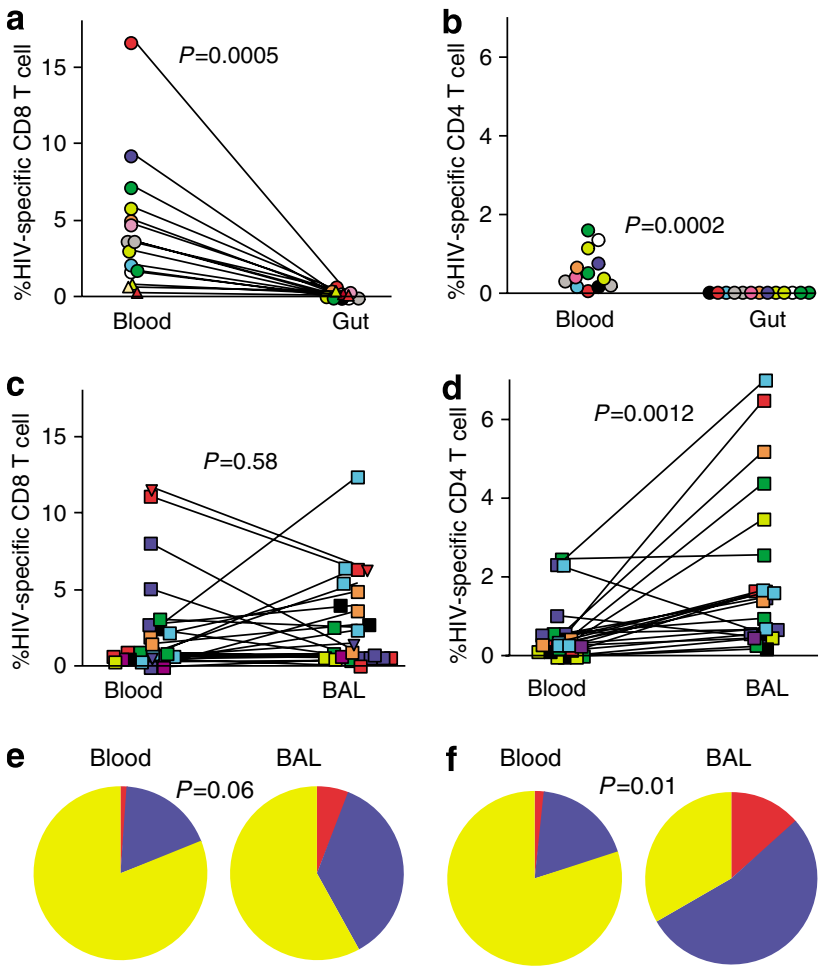

$\square$ function

2 function

1 function

Figure 5 Frequency and functionality of HIV-specific T cells in blood, GI tract, and BAL. (a) HIV-specific CD8 T-cell responses in blood and gut. (b) HIV-specific CD4 T-cell responses in blood and gut. (c) HIVspecific CD8 T-cell responses in blood and BAL. (d) HIV-specific CD4 T-cell responses in blood and BAL. (e) Functionality of HIV-specific CD8 and (f) CD4 T cells. Lymphocytes were stimulated with overlapping HIV peptides and stained as described in Methods. The responses are shown as the percentage of memory T cells that are HIV specific. Circles or squares denote individuals' responses, with some individuals responding to multiple peptide pools as denoted with multiple like-colored symbols. In addition, tetramer analysis was performed on several individuals, and the frequencies of memory CD8 T cells that bind tetramer are denoted by triangles. Points depicting values for the same individual are linked by a line. $P$-values represent the results of Wilcoxon's matched pairs test. The frequency of the total HIV-specific responses producing one, two, or three cytokines was determined using SPICE as described in Methods. $\mathrm{BAL}$, bronchoalveolar lavage; GI, gastrointestinal.

HIV-specific T cells and increased levels of CCR5-binding chemokines are associated with preservation of local CD4 $\mathrm{T}$ cells.

There are at least two explanations that might account for the diametrically opposed immunological outcomes that we observed at different mucosal sites. First, the terminal ileum could be so profoundly damaged during the acute phase of infection by the virus itself that subsequent immunological events, such as initiation of an HIV-specific immune response and CD4 T-cell reconstitution, are hindered. Second, local HIV-specific immune responses within individual mucosal sites may suppress viral replication, thereby inhibiting viral infection and preserving memory CD4 T cells.

In support of the first scenario, recent data have demonstrated that the majority of CD4 T cells in the GI tract are
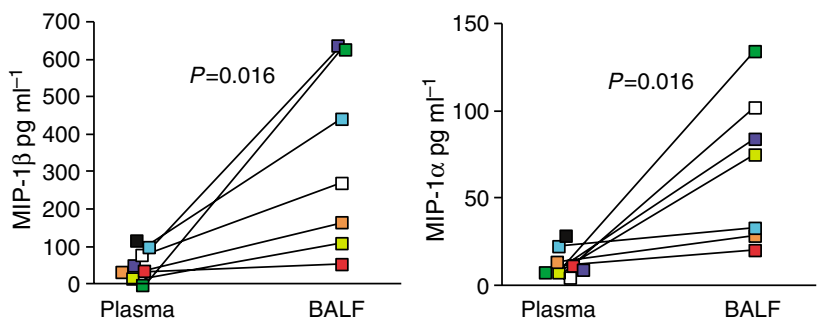

Figure 6 MIP- $1 \alpha$ and MIP- $1 \beta$ levels in BAL and plasma. BAL fluid and cells were collected during lavage as described in Methods. Acellular BAL fluid was concentrated $4 \times$ and stored frozen until chemokine analysis. Measurements of chemokines in BAL fluid were adjusted for dilution by the urea method. Chemokines were measured by cytokine bead array on a FACSCalibur. Points depicting values for the same individual are linked by a line. BAL, bronchoalveolar lavage.

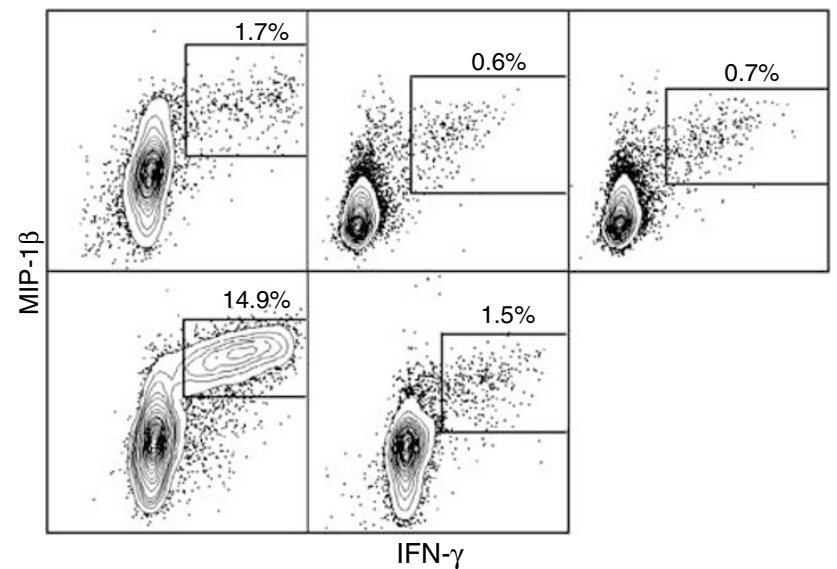

Figure 7 MIP- $1 \beta$ production by HIV-specific CD8 T cells in BAL. BAL lymphocytes were stimulated with overlapping HIV peptides and stained as described in Methods with the exception that anti-MIP-1 $\beta$ PE was substituted for anti-CD27 PE. Gated live CD8 T cells are shown and all IFN- $\gamma$ also express MIP-1 $\beta$. PE, phycoerythrin.

directly infected by the virus or die as a result of apoptosis during the acute phase of SIV infection in rhesus macaques and subsequently depleted. ${ }^{4,5}$ Similar levels of depletion are also observed in HIV-infected individuals $\mathrm{s}^{2,3,6}$ and high frequencies of infection within GI tract CD4 $\mathrm{T}$ cells are also observed during acute HIV infection. ${ }^{31}$ Here, we show further that GI tract CD4 T-cell depletion is associated with high frequencies of infected CD4 T cells during all stages of infection; this finding indicates that the reservoir of infected CD4 $\mathrm{T}$ cells is substantially larger than analysis of peripheral blood CD4 T cells alone would suggest. Moreover, GI tract CD4 T cells do not appear to reconstitute substantially with long-term highly active antiretroviral therapy even though blood CD4 T cells recover to a variable extent. ${ }^{32}$ Hence, there are precedent data to suggest that virusinduced damage to the GI tract during the acute phase of infection renders the GI tract incapable of supporting subsequent healthy immunological processes. These findings might explain the paucity of HIV-specific T-cell responses we observe within the terminal ileum. The lack of comparably high levels of viral replication within other mucosal sites may thus allow for preservation of local CD4 T cells and the successful induction and 
maintenance of HIV-specific T-cell responses. Consistent with this explanation, HIV-specific T cells have been detected in rectal biopsies of HIV-infected humans, ${ }^{12-14,33}$ and CD4 T-cell depletion in the colon may be less severe than depletion in the small intestine. ${ }^{13,33}$ In addition, Mamu ${\mathrm{A} 01^{+}}^{+}$rhesus macaques attain significantly lower set point VLs after SIV infection compared to rhesus macaques that do not express the A01 allele, ${ }^{34}$ and these monkeys manifest high frequencies of Mamu-A01restricted SIV-specific CD8 T cells in both small and large intestines. ${ }^{35-37}$ Moreover, Mamu-A01 ${ }^{+}$rhesus macaques that fail to control viral replication lack functional SIV-specific CD8 T cells in mucosal sites. 35

Alternatively, local HIV-specific T-cell responses in BAL may suppress viral replication by direct anti-viral effector mechanisms including cytolysis of infected cells and secretion of antiviral chemokines and cytokines. While early studies of BAL lymphocytes suggested that the presence of cytotoxic HIV-specific CD8 T cells was associated with poor clinical outcome, ${ }^{38-42}$ we found functional HIV-specific CD8 T cells in the BAL of all HIV-infected individuals we studied. Indeed, the local presence of polyfunctional HIV-specific T cells and the CCR5-binding chemokines, which they may produce, was associated with an apparent protection against high frequencies of HIV infection and massive depletion of mucosal CD4 T cells.

In summary, while causal relationships between viral replication and HIV-specific immune responses are difficult to ascertain, our findings show an anatomically distinct mucosal site that contains ample cellular targets for HIV infection, which nevertheless remains relatively intact and which also harbors robust HIV-specific T-cell immunity. These findings thus provide a certain degree of optimism that vaccines aimed at eliciting mucosal HIV-specific T cells might attenuate GI tract CD4 $\mathrm{T}$-cell depletion. Indeed, such vaccines show some promise in SIV infection of rhesus macaques. ${ }^{15,16,43}$ Moreover, these data provide additional rationale for the use of currently evolving therapeutic CCR5 antagonists. ${ }^{44}$

\section{METHODS}

Subjects. Twenty-six antiretroviral therapy-naive HIV-infected and 12 HIV-uninfected subjects were recruited for this study. Clinical details are shown in Table $\mathbf{1}$ and subjects are coded by color and shape in all figures. $\mathrm{HIV}^{+}$individuals were classified as being "early" based on HIV seropositivity for less than 1 year with maintained CD4 T-cell counts of greater than $200 \mathrm{CD} 4 \mathrm{~T}$ cells per $\mu \mathrm{l}$, "chronic" based on seropositivity for greater than 1 year with CD4 counts above 200, and "AIDS" based on a CD4 count of less than 200. VL was determined using either the Roche Amplicor Monitor assay or the Roche Ultradirect assay. All subjects gave informed consent in compliance with the appropriate institutional review boards.

Samples. Peripheral blood mononuclear cells were prepared from venous blood by density gradient centrifugation. Ileal Peyer's patches and lamina propria samples were acquired by endoscopy and biopsy of the terminal ileum. Patients received standard colonoscopy preparation, GoLYTELY (Braintree Laboratories, Braintree, MA) and were sedated with Versed and Fentanyl. A colonoscope was passed through the large intestine and through the ileo-cecal valve. At least 8-10 biopsy samples were obtained with cold forceps using standard techniques. Biopsies were placed in RPMI media supplemented with $10 \%$ heat-inactivated fetal calf serum (R10) and shipped on wet ice overnight for T-cell analysis. GI tract samples were then dissected into $100 \mu \mathrm{l}$ fragments and were digested in Isocove's media supplemented with $2 \mathrm{mg} \mathrm{ml}^{-1}$ type 2 collagenase (Sigma, St Louis, MO) and $1 \mathrm{U} \mathrm{ml}^{-1} \mathrm{DNase} \mathrm{I}$ (Sigma) for $30 \mathrm{~min}$ at $37^{\circ} \mathrm{C}$. Following digestion, samples were passed through a $100 \mu \mathrm{m}$ filter and were washed twice with R10. From this procedure, approximately $3 \times 10^{6}$ live lymphocytes are routinely isolated. BAL cells were obtained after anesthetizing the upper airways with $4 \%$ lidocaine nebulizer and the vocal cords and proximal airways with $1 \%$ lidocaine solution. Bronchoscopy with lavage was performed through a fiberoptic bronchoscope wedged in a subsegmental bronchus of the right middle and right lower lobes as previously described. ${ }^{40} \mathrm{~A}$ volume of $150 \mathrm{ml}$ of normal saline at room temperature was instilled in 50-ml aliquots into the medial segment of the right middle lobe and repeated in the anterior segment of the right lower lobe. Subjects were sedated with stadol and midazolam. Typically, $300 \mathrm{ml}$ was instilled to obtain a return of $125-200 \mathrm{ml}$ of BALF. BALF was filtered through 100- $\mu$ m Nylon mesh (Tetko, Elmsford, NY) to remove debris, and was centrifuged at $400 \mathrm{~g}$ for $10 \mathrm{~min}$. Recovered BALF was concentrated $4 \times$ by filter centrifugation and BALF was normalized by the urea method as previously described. ${ }^{27} \mathrm{BALF}$ was then frozen at $-70^{\circ} \mathrm{C}$ in small aliquots for chemokine and VL determination. The cell pellet was washed twice and resuspended in R10. Suspended cells were then shipped on wet ice overnight for T-cell analysis. Comparative studies of fresh vs. 1-day-old BAL lymphocytes revealed that overnight shipping did not dramatically affect lymphocyte viability and had no affect on the frequencies of individual T-cell subsets. Cell differentials were obtained from cytospin slides.

Flow cytometric analysis. Eighteen-parameter flow cytometric analysis was performed using a FACSAira flow cytometer (Becton Dickinson, San Jose, CA). Fluorescein isothiocyanate, phycoerythrin (PE), Cy7PE, Cy5.5PE, allophycocyanin (APC), Cy7APC, Texas Red PE, violet amine reactive dye, and Quantum-dot 705 (QD705) were used as fluorophores. At least 300,000 live lymphocytes were collected. The list-mode data files were analyzed using FlowJo (Tree Star, Ashland, OR). Functional capacity was determined after Boolean gating and subsequent analysis was performed using Simplified Presentation of Incredibly Complex Evaluations (SPICE, version 2.9, Mario Roederer, VRC, NIAID, NIH). All values used for analyzing proportionate representation of responses are background-subtracted.

HIV-specific T-cell assay. Stimulation was performed on fresh or frozen lymphocytes as described elsewhere ${ }^{45}$ Freshly isolated or freshly thawed lymphocytes were resuspended at $10^{6} \mathrm{ml}^{-1}$ in $\mathrm{R} 10$ supplemented with $1 \mu \mathrm{g} \mathrm{ml}^{-1}$ anti-CD28 and anti-CD49d antibodies. Peptides 15 amino acids in length, overlapping by 11 amino acids, and encompassing HIV$1 \mathrm{Gag}$, Pol, Nef, and Env (corresponding to the sequence of HXBc2) were used to stimulate HIV-specific T cells in the presence of Brefeldin-A (BFA, $1 \mu \mathrm{g} \mathrm{ml}^{-1}$; Sigma) for $18 \mathrm{~h}$ at $37^{\circ} \mathrm{C}$. All cells were surface-stained for phenotypic markers of interest and intracellularly stained for cytokines (intracellular cytokine staining).

Monoclonal antibodies, tetrameric complexes, and T-cell phenotyping. Monoclonal antibodies used for phenotypic and functional characterization of T-cell subsets were anti-CD3 Cy7APC, anti-CD45RO Texas Red PE, anti-CD27 PE (or CCR5 PE), anti-CD4 Cy5.5PE, anti-CD8 QD705, anti-IFN $\gamma$ fluorescein isothiocyanate, and anti-TNF Cy7PE and anti-IL-2 APC (Becton Dickinson Pharmingen, San Diego, CA). As naive T cells do not express CCR5, and as HIV-specific T cells are not detectable in the naive T-cell pool, we report these data as percentages of memory $\mathrm{T}$ cells. We first gated for memory CD4 and CD8 T cells based on characteristic expression patterns of CD45RO and CD27. We then determined the percentage of CD4 and CD8 T cells that express CCR5 or are HIV specific. Tetrameric complexes used to examine the frequency of $\mathrm{HIV}$-specific $\mathrm{CD}^{+}$ T cells were HLA-B57 KAFSPEVIPMF, HLA-A2 SLYNTVATL, HLA-B8 GGKKKYKL, and HLA-B8 FLKEKGGL, all prepared using strepavidin APC (Prozyme, San Leonardo, CA) as described previously. ${ }^{46}$ 
CCR5 ligand analysis. Plasma samples were diluted 1:4 and BALs were concentrated $4 \times$ by filter centrifugation (Millipore, Billerica, MA) and BALF was normalized by the urea method as previously described. ${ }^{27}$ Samples were then run using Cytokine Bead Array (CBA, BD) according to the manufacturer's protocol. Standards were prepared and a twofold serial dilution was performed. Fifty microliters of unknown or standard was incubated with cytokine-specific antibody-conjugated beads and incubated for $1 \mathrm{~h}$ at room temperature. A PE detection reagent was added and the incubation continued a further $2 \mathrm{~h}$ at room temperature in the dark. Samples were then washed and resuspended in buffer. Samples were analyzed immediately on a FACSCalibur flow cytometer and data were analyzed with either the Cytokine Bead Array analysis program in Excel or FCAP Array.

Quantitative PCR. Quantification of HIV gag DNA in sorted memory CD4 T cells was performed by qPCR by means of the 5 ' nuclease (TaqMan) assay with an ABI7700 system (PerkinElmer, Norwalk, CT) as previously described. ${ }^{22,47}$ To quantify cell number in each reaction, qPCR was performed simultaneously for albumin gene copy number as previously described ${ }^{48}$ Standards were constructed for absolute quantification of gag and albumin copy number, and were validated with sequential dilutions of $8 \mathrm{E} 5$ cell lysates that contain one copy of gag per cell. Duplicate reactions were run and template copies calculated using ABI7700 software. CCR5 mRNA levels were normalized to mRNA levels of the cellular gene H-Gus. Results are plotted as the ratio of CCR5 mRNA levels in BAL AM compared to levels in BAL T cells.

\section{ACKNOWLEDGMENTS}

We thank Drs Rhame, Lifson, Kline, Simpson, Baker, Gupta, and Goldman for patient referral and the Indiana University GCRC for patient care. DAP is a Medical Research Council (UK) Senior Clinical Fellow. KSK is supported by NIH Grants K08HL04545-05 and R01 HL083468. HLT is supported by NIH Grant R01HL59834. TWS is supported by NIH Grants R01 Al54232, K24 Al056986, and R01 DE-12934. This work was supported, in part, by intramural funding from NIAID, NIH.

\section{DISCLOSURE}

The authors declared no conflict of interest.

\section{2008 Society for Mucosal Immunology}

\section{REFERENCES}

1. Veazey, R.S., DeMaria, M., Chalifoux, L.V., Shvetz, D.E., Pauley, D.R. et al. Gastrointestinal tract as a major site of CD4+ T cell depletion and viral replication in SIV infection. Science 280, 427-431 (1998).

2. Brenchley, J.M., Schacker, T.W., Ruff, L.E., Price, D.A., Taylor, J.H et al. $\mathrm{CD} 4+\mathrm{T}$ cell depletion during all stages of HIV disease occurs predominantly in the gastrointestinal tract. J. Exp. Med. 200, 749-759 (2004).

3. Mehandru, S., Poles, M.A., Tenner-Racz, K., Horowitz, A., Hurley, A. et al. Primary HIV-1 infection is associated with preferential depletion of CD4+ T lymphocytes from effector sites in the gastrointestinal tract. J. Exp. Med. 200, 761-770 (2004).

4. Mattapallil, J.J., Douek, D.C., Hill, B., Nishimura, Y., Martin, M. et al. Massive infection and loss of memory CD4+ T cells in multiple tissues during acute SIV infection. Nature 434, 1093-1097 (2005).

5. Li, Q., Duan, L., Estes, J.D., Ma, Z.M., Rourke, T. et al. Peak SIV replication in resting memory $C D 4+T$ cells depletes gut lamina propria CD4+ T cells. Nature 434, 1148-1152 (2005).

6. Guadalupe, M., Reay, E., Sankaran, S., Prindiville, T., Flamm, J. et al. Severe CD4+ T-cell depletion in gut lymphoid tissue during primary human immunodeficiency virus type 1 infection and substantial delay in restoration following highly active antiretroviral therapy. J. Virol. 77, 11708-11717 (2003).

7. Lifson, J.D., Rossio, J.L., Piatak, M. Jr., Parks, T., Li, L. et al. Role of CD8(+) lymphocytes in control of simian immunodeficiency virus infection and resistance to rechallenge after transient early antiretroviral treatment. J. Virol. 75, 10187-10199 (2001).
8. Koup, R.A., Safrit, J.T., Cao, Y., Andrews, C.A., Wu, Y. et al. Temporal association of cellular immune response with the initial control of viremia in primary HIV-1 syndrome. J. Virol. 68, 4650-4655 (1994).

9. Price, D.A., Goulder, P.J., Klenerman, P., Sewell, A.K., Easterbrook, P.J. et al. Positive selection of HIV-1 cytotoxic T lymphocyte escape variants during primary infection. Proc. Natl. Acad. Sci. USA 94, 1890-1895 (1997).

10. Martin, M.P. \& Carrington, M. Immunogenetics of viral infections. Curr. Opin. Immunol. 17, 510-516 (2005).

11. Sewell, A.K., Price, D.A., Oxenius, A., Kelleher, A.D. \& Phillips, R.E. Cytotoxic T lymphocyte responses to human immunodeficiency virus: control and escape. Stem Cells 18, 230-244 (2000).

12. Musey, L., Ding, Y., Cao, J., Lee, J., Galloway, C. et al. Ontogeny and specificities of mucosal and blood human immunodeficiency virus type 1specific CD8(+) cytotoxic T lymphocytes. J. Virol. 77, 291-300 (2003).

13. Shacklett, B.L., Cox, C.A., Sandberg, J.K., Stollman, N.H., Jacobson, MA. et al. Trafficking of human immunodeficiency virus type 1-specific CD8+ T cells to gut-associated lymphoid tissue during chronic infection. J. Virol. 77, 5621-5631 (2003).

14. Ibarrondo, F.J., Anton, P.A., Fuerst, M., Ng. H.L., Wong, J.T. et al. Parallel human immunodeficiency virus type 1-specific CD8+ T-lymphocyte responses in blood and mucosa during chronic infection. J. Virol. 79, 4289-4297 (2005).

15. Mattapallil, J.J., Douek, D.C., Buckler-White, A., Montefiori, D., Letvin, N.X. et al. Vaccination preserves CD4 memory T cells during acute simian immunodeficiency virus challenge. J. Exp. Med. 203, 1533-1541 (2006).

16. Letvin, N.L., Mascola, J.R., Sun, Y., Gorgone, D.A., Buzby, A.P. et al. Preserved CD4+ central memory T cells and survival in vaccinated SIVchallenged monkeys. Science 312, 1530-1533 (2006).

17. Wilson, N.A., Reed, J., Napoe, G.S., Piaskowski, S., Szymanski, A. et al. Vaccine-induced cellular immune responses reduce plasma viral concentrations after repeated low-dose challenge with pathogenic simian immunodeficiency virus SIVmac239. J. Virol. 80, 5875-5885 (2006).

18. Lefrancois, L. \& Puddington, L. Intestinal and pulmonary mucosal T cells: local heroes fight to maintain the status quo. Annu. Rev. Immunol. 24, 681-704 (2006)

19. Ancochea, J., Gonzalez, A., Sanchez, M.J., Aspa, J. \& Lopez-Botet, M. Expression of lymphocyte activation surface antigens in bronchoalveolar lavage and peripheral blood cells from young healthy subjects. Chest 104, 32-37 (1993).

20. Costabel, U., Bross, K.J., Marxen, J. \& Matthys, H. T-lymphocytosis in bronchoalveolar lavage fluid of hypersensitivity pneumonitis. Changes in profile of T-cell subsets during the course of disease. Chest 85, 514-522 (1984).

21. Myou, S., Fujimura, M., Yasui, M., Ueno, T. \& Matsuda, T. Bronchoalveolar lavage cell analysis in measles viral pneumonia. Eur. Respir. J. 6, 1437-1442 (1993).

22. Brenchley, J.M., Hill, B.J., Ambrozak, D.R., Price, D.A., Guenaga, F.J. et al. T-cell subsets that harbor human immunodeficiency virus (HIV) in vivo: implications for HIV pathogenesis. J. Virol. 78, 1160-1168 (2004).

23. Toossi, Z., Nicolacakis, K., Xia, L., Ferrari, N.A. \& Rich, E.A. Activation of latent HIV-1 by Mycobacterium tuberculosis and its purified protein derivative in alveolar macrophages from HIV-infected individuals in vitro. J. Acquir. Immune Defic. Syndr. Hum. Retrovirol. 15, 325-331 (1997).

24. Lewin, S.R., Kirihara, J., Sonza, S., Irving, L., Mills, J. et al. HIV-1 DNA and mRNA concentrations are similar in peripheral blood monocytes and alveolar macrophages in HIV-1-infected individuals. AIDS 12, 719-727 (1998).

25. Barber, S.A., Gama, L., Li, M., Voelker, T., Anderson, J.E. et al. Longitudinal analysis of simian immunodeficiency virus (SIV) replication in the lungs: compartmentalized regulation of SIV. J. Infect. Dis. 194, 931-938 (2006).

26. Betts, M.R., Nason, M.C., West, S.M., De Rosa, S.C., Migueles, S.A. et al. HIV nonprogressors preferentially maintain highly functional HIVspecific CD8+ T-cells. Blood 107, 4781-4789 (2006).

27. Rennard, S.I., Basset, G., Lecossier, D., O'Donnell, K.M., Pinkston, P. et al. Estimation of volume of epithelial lining fluid recovered by lavage using urea as marker of dilution. J. Appl. Physiol. 60, 532-538 (1986).

28. Cocchi, F., DeVico, A.L., Garzino-Demo, A., Arya, S.K., Gallo, R.C. et al. Identification of RANTES MIP-1 alpha, and MIP-1 beta as the major HIVsuppressive factors produced by CD8+ T cells. Science $\mathbf{2 7 0 , 1 8 1 1 - 1 8 1 5}$ (1995). 
29. Price, D.A., Sewell, A.X., Dong, T., Tan, R., Goulder, P.J. et al. Antigenspecific release of beta-chemokines by anti-HIV-1 cytotoxic $T$ lymphocytes. Curr. Biol. 8, 355-358 (1998).

30. Meschi, J., Crouch, B.C., Skolnik, P., Yahya, K., Holmskov, U. et al. Surfactant protein D binds to human immunodeficiency virus (HIV) envelope protein gp120 and inhibits HIV replication. J. Gen. Virol. 86, 3097-3107 (2005).

31. Mehandru, S., Poles, M.A., Tenner-Racz, K., Manuelli, V., Jean-Pierre, P. et al. Mechanisms of gastrointestinal CD4+ T-cell depletion during acute and early human immunodeficiency virus type 1 infection. J. Virol. 81, 599-612 (2007).

32. Guadalupe, M., Sankaran, S., George, M.D., Reay, E., Verhoeven, D. et al. Viral suppression and immune restoration in the gastrointestinal mucosa of human immunodeficiency virus type 1-infected patients initiating therapy during primary or chronic infection. J. Virol. 80, 8236-8247 (2006)

33. Critchfield, J.W., Lemongello, D., Walker, D.H., Garcia, J.C., Asmuth, D.M. et al. Multifunctional HIVgag specific CD8+ T-cell responses in rectal mucosa and PBMC during chronic HIV-1 infection. J. Virol. 81, 5460-5471 (2007).

34. Mothe, B.R., Weinfurter, J., Wang, C., Rehrauer, W., Wilson, N. et al. Expression of the major histocompatibility complex class I molecule Mamu- $A^{*} 01$ is associated with control of simian immunodeficiency virus SIVmac239 replication. J. Virol. 77, 2736-2740 (2003).

35. Hel, Z., Nacsa, J., Kelsall, B., Tsai, W.P., Letvin, N. et al. Impairment of Gag-specific CD8(+) T-cell function in mucosal and systemic compartments of simian immunodeficiency virus mac251- and simianhuman immunodeficiency virus KU2-infected macaques. J. Virol. 75, 11483-11495 (2001).

36. Veazey, R.S., Lifson, J.D., Schmitz, J.E., Kuroda, M.J., Piatak, M. Jr. et al. Dynamics of Simian immunodeficiency virus-specific cytotoxic T-cell responses in tissues. J. Med. Primatol. 32, 194-200 (2003).

37. Schmitz, J.E., Veazey, R.S., Kuroda, M.J., Levy, D.B., Seth, A. et al. Simian immunodeficiency virus (SIV)-specific cytotoxic T lymphocytes in gastrointestinal tissues of chronically SIV-infected rhesus monkeys. Blood 98, 3757-3761 (2001).

38. Guillon, J.M., Autran, B., Denis, M., Fouret, P., Plata, F. et al. Human immunodeficiency virus-related lymphocytic alveolitis. Chest $\mathbf{9 4}$, 1264-1270 (1988).
39. Plata, F., Autran, B., Martins, L.R., Wain-Hobson, S., Raphael, M. et al. AIDS virus-specific cytotoxic T lymphocytes in lung disorders. Nature 328, 348-351 (1987)

40. Twigg, H.L., Soliman, D.M., Day, R.B., Knox, K.S., Anderson, R.J. et al. Lymphocytic alveolitis, bronchoalveolar lavage viral load, and outcome in human immunodeficiency virus infection. Am. J. Respir. Crit. Care Med. 159, 1439-1444 (1999).

41. Agostini, C., Zambello, R., Trentin, L., Poletti, V., Spiga, L. et al. Prognostic significance of the evaluation of bronchoalveolar lavage cell populations in patients with HIV-1 infection and pulmonary involvement. Chest 100, 1601-1606 (1991).

42. Agostini, C., Poletti, V., Zambello, R., Trentin, L., Siviero, F. et al. Phenotypical and functional analysis of bronchoalveolar lavage lymphocytes in patients with HIV infection. Am. Rev. Respir. Dis. 138, 1609-1615 (1988).

43. Mattapallil, J.J., Hill, B., Douek, D.C. \& Roederer, M. Systemic vaccination prevents the total destruction of mucosal CD4 T cells during acute SIV challenge. J. Med. Primatol. 35, 217-224 (2006).

44. Veazey, R.S., Klasse, P.J., Ketas, T.J., Reeves, J.D., Piatak, R. Jr. et al. Use of a small molecule CCR5 inhibitor in macaques to treat simian immunodeficiency virus infection or prevent simian-human immunodeficiency virus infection. J. Exp. Med. 198, 1551-1562 (2003).

45. Pitcher, C.J., Quittner, C., Peterson, D.M., Connors, M., Koup, R.A. et al. HIV-1-specific CD4+ T cells are detectable in most individuals with active HIV-1 infection, but decline with prolonged viral suppression. Nat. Med. 5, 518-525 (1999).

46. Hutchinson, S.L., Wooldridge, L., Tafuro, S., Lauge, L.B., Glick, M. et al. The CD8 T cell coreceptor exhibits disproportionate biological activity at extremely low binding affinities. J. Biol. Chem. 278, 24285-24293 (2003).

47. Douek, D.C., Berts, M.R., Brenchley, J.M., Hill, B.I., Ambrozak, D.R. et al. A novel approach to the analysis of specificity, clonality, and frequency of HIV-specific T cell responses reveals a potential mechanism for control of viral escape. J. Immunol. 168, 3099-3104 (2002).

48. Douek, D.C., Brenchley, J.M., Betts, M.R., Ambrozak, D.R., Hill, B.J. et al HIV preferentially infects HIV-specific CD4+ T-cells. Nature 417, 95-98 (2002). 